TRANSACTIONS OF THE

AMERICAN MATHEMATICAL SOCIETY

Volume 359, Number 3, March 2007, Pages 1115-1128

S 0002-9947(06)04271-1

Article electronically published on July 21, 2006

\title{
COUNTEREXAMPLES TO THE POSET CONJECTURES OF NEGGERS, STANLEY, AND STEMBRIDGE
}

\author{
JOHN R. STEMBRIDGE
}

\begin{abstract}
We provide the first counterexamples to Neggers' 1978 conjecture and Stembridge's 1997 conjecture that the generating functions for descents and peaks in the linear extensions of naturally labeled posets should have all real zeros. We also provide minimum-sized counterexamples to a generalization of the Neggers conjecture due to Stanley that was recently disproved by Brändén.
\end{abstract}

\section{INTRODUCTION}

Given a partial ordering $P$ of $[n]=\{1, \ldots, n\}$, let $\mathcal{L}(P)$ denote the set of linear extensions of $P$; i.e., the set of permutations $w=\left(w_{1}, \ldots, w_{n}\right)$ of $[n]$ such that if $i \prec j$ in $P$, then $i$ precedes $j$ in $w$. For each linear extension $w$, we let

$$
\begin{aligned}
d(w) & :=\left|\left\{i: w_{i}>w_{i+1}\right\}\right|, \\
\Lambda(w) & :=\left|\left\{i: w_{i-1}<w_{i}>w_{i+1}\right\}\right|
\end{aligned}
$$

denote the number of descents and peaks in $w$, respectively. (Note that we use " $\prec$ " for the order relation of $P$, whereas " $<$ " is reserved for the usual total ordering of the integers.)

We say that $P$ is naturally labeled if $i \prec j$ implies $i<j$ for all $i$ and $j$.

In 1978, Neggers [N] conjectured that if $P$ is naturally labeled, then the polynomial

$$
W_{P}(t):=\sum_{w \in \mathcal{L}(P)} t^{d(w)+1}
$$

should have all real zeros. Subsequently in 1986, Stanley conjectured that the hypothesis of natural labeling should be unnecessary; i.e., that the $W$-polynomial of every partial ordering of $[n]$ should have all real zeros. This conjecture seems to have been first mentioned in print by Brenti $\mathrm{Br}$.

The conjectures of Neggers and Stanley have attracted widespread attention, including numerous papers providing partial or supporting results (e.g., see B1, [B2, $\mathrm{Br}, \mathrm{G}, \mathrm{RW},[\mathrm{Si}$, and $\mathrm{W}]$ ). Also, in 1997 we developed a theory of "enriched $P$-partitions" St in which the distribution of peaks in $\mathcal{L}(P)$, and a variation of the $W$-polynomial, viz.,

$$
\bar{W}_{P}(t):=\sum_{w \in \mathcal{L}(P)} t^{\Lambda(w)+1}
$$

Received by the editors December 6, 2004.

2000 Mathematics Subject Classification. Primary 06A07, 06-04, 05A15.

This work was supported by NSF grant DMS-0245385.

(C)2006 American Mathematical Society Reverts to public domain 28 years from publication 


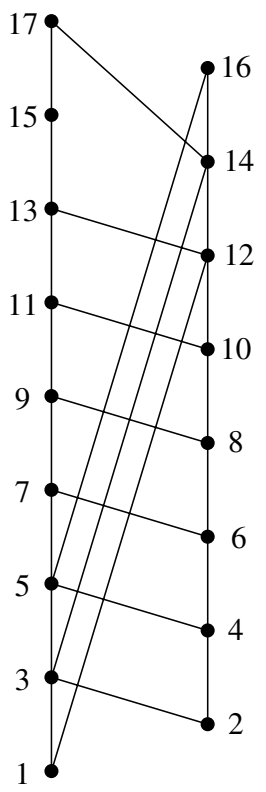

Figure 1

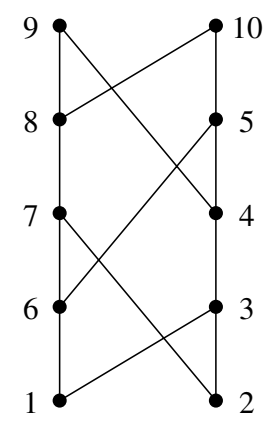

FIGURE 2

play a significant role. In particular, we conjectured that all zeros of $\bar{W}_{P}(t)$ should be real, and confirmed this conjecture and the Neggers-Stanley conjecture for all partial orderings on $\leqslant 7$ vertices, and all natural partial orderings on 8 vertices.

However, Petter Brändén recently discovered a family of counterexamples to Stanley's conjecture [B3]. Specifically, the posets $P=P_{m, n}$ consisting of the two chains

$$
1 \prec 2 \prec \cdots \prec m, \quad m+1 \prec m+2 \prec \cdots \prec m+n,
$$

together with the "unnatural" relation $m+1 \prec m$ produce $W$-polynomials with nonreal zeros provided that $m$ and $n$ are sufficiently large. In particular, Brändén notes that $P_{11,11}$ has this property.

These counterexamples inspired us to conduct a systematic computer search for $W$-polynomials with nonreal zeros among the partially ordered sets that are narrow in the sense that their vertices may be partitioned into two chains. Note that by Dilworth's Theorem, a poset is narrow if and only if it has no antichain of 3 elements.

Our search has revealed that there exist naturally labeled narrow posets on 17 vertices whose $W$-polynomials have nonreal zeros (and none smaller), thereby disproving Neggers' original conjecture. For example, the $W$-polynomial of the poset in Figure 1 is

$$
t+32 t^{2}+336 t^{3}+1420 t^{4}+2534 t^{5}+1946 t^{6}+658 t^{7}+86 t^{8}+3 t^{9},
$$

and this polynomial has a conjugate pair of zeros near $t=-1.858844 \pm 0.149768 i$.

In a second search, we discovered that the smallest narrow counterexamples for Stanley's conjecture (i.e., arbitrarily labeled posets whose $W$-polynomials have nonreal zeros) have 10 vertices. For example, the $W$-polynomial of the poset in Figure 2 is

$$
11 t^{2}+42 t^{3}+50 t^{4}+18 t^{5}+t^{6}
$$


and this polynomial has a pair of zeros near $t=-0.614039 \pm 0.044227 i$.

It would be interesting to know if the narrow counterexamples we have found are the smallest counterexamples among all posets. In this direction, we have confirmed by computer search that there are no counterexamples to the Stanley conjecture with $\leqslant 9$ vertices, so the 10 -vertex counterexamples are minimal among all posets, but there could be other 10-vertex counterexamples that are not narrow. Furthermore, we have also checked that there are no counterexamples to the Neggers conjecture on $\leqslant 10$ vertices. Note that there are roughly $4.7 \times 10^{7}$ isomorphism classes of posets with 11 vertices, and $4.5 \times 10^{15}$ with 16 vertices (for exact counts, see the work of Brinkmann and McKay $[\mathrm{BM}]$ ).

Turning now to $\bar{W}$-polynomials, let $P^{+}$denote the partial ordering of $[n+1]$ obtained by adjoining 0 to $P$ as a minimum element, and then shifting the vertex labels by 1 .

Proposition 1.1. If the vertices of $P$ can be partitioned into two naturally labeled chains, then $\bar{W}_{P^{+}}(t)=W_{P^{+}}(t)=W_{P}(t)$.

Proof. The map $w \mapsto w^{+}=\left(1, w_{1}+1, \ldots, w_{n}+1\right)$ is a bijection from $\mathcal{L}(P)$ to $\mathcal{L}\left(P^{+}\right)$. Given a partition of $P$ into two naturally labeled chains, every $w \in \mathcal{L}(P)$ may be partitioned into two increasing subsequences. Every descent in $w^{+}$is therefore preceded by an ascent (in particular, note that there is always an ascent in the first position), and thus participates in a peak. Hence, $\Lambda\left(w^{+}\right)=d\left(w^{+}\right)=d(w)$ for all $w \in \mathcal{L}(P)$.

It is easy to see that the posets in Figures 1 and 2, as well as Brändén's posets $P_{m, n}$, can each be partitioned into two naturally labeled chains. Thus, by adjoining minimum elements, one may produce an 11-vertex counterexample and an 18-vertex naturally labeled counterexample to our 1997 conjecture that all zeros of $\bar{W}$-polynomials should be real. We have also confirmed by computer search that these counterexamples are of minimum size within their respective categories (i.e., narrow posets and naturally labeled narrow posets whose $\bar{W}$-polynomials have nonreal zeros).

In the remainder of this paper, we present an algorithm for computing $W$ polynomials and $\bar{W}$-polynomials that runs in polynomial time on narrow posets (Section 2), introduce a subtle equivalence relation ("shift-equivalence") on partial orderings of $[n]$ that preserves the $W$-polynomial up to powers of $t$ (Section 4 ), describe some special structural features that simplify the problem of efficiently searching through isomorphism classes of narrow posets (Section 5), and provide more details about the search results (Section 6). In the final section, we discuss possible directions for future research in this area.

The programs we used to conduct the searches, as well as detailed listings of the results, are available at: www.math. Isa.umich.edu/ jrs/data/pocon/.

\section{ORDER POLYNOMIALS AND EQUIVALENCE OF LABELINGS}

The order polynomial of the poset $P$, denoted $\Omega_{P}$, may be defined by the property that for integers $m \geqslant 0, \Omega_{P}(m)$ is the number of maps $f: P \rightarrow[m]$ such that

$$
\begin{gathered}
i \preccurlyeq j \Rightarrow f(i) \leqslant f(j), \\
i \prec j \text { and } i>j \Rightarrow f(i) \neq f(j) .
\end{gathered}
$$


Similarly, the enriched order polynomial of $P$, denoted $\bar{\Omega}_{P}$, may be defined by the property that $\bar{\Omega}_{P}(m)$ is the number of maps $f: P \rightarrow[2 m]$ such that

$$
\begin{gathered}
i \preccurlyeq j \Rightarrow f(i) \leqslant f(j), \\
i \prec j, i<j \text { and } f(i)=f(j) \Rightarrow f(i) \text { is even, } \\
i \prec j, i>j \text { and } f(i)=f(j) \Rightarrow f(i) \text { is odd. }
\end{gathered}
$$

Among the basic consequences of the theory of $P$-partitions are the identities

$$
\begin{aligned}
& \sum_{m \geqslant 0} \Omega_{P}(m) t^{m}=\frac{1}{(1-t)^{n+1}} \cdot W_{P}(t), \\
& \sum_{m \geqslant 0} \bar{\Omega}_{P}(m) t^{m}=\frac{1}{2}\left(\frac{1+t}{1-t}\right)^{n+1} \cdot \bar{W}_{P}\left(\frac{4 t}{(1+t)^{2}}\right) .
\end{aligned}
$$

The first of these is due to Stanley [S] the second is due to Stembridge [St].

These identities are important for both conceptual and computational reasons.

We declare a partial ordering $Q$ of $[n]$ to be equivalent to $P$ if there is a poset isomorphism $\pi: P \rightarrow Q$ such that for each covering relation $i \prec j$ in $P$, we have

$$
i<j \Leftrightarrow \pi(i)<\pi(j) .
$$

In particular, all natural labelings in the isomorphism class of $P$ are equivalent.

It is clear from the definitions that if $P$ and $Q$ are equivalent, then they have the same ordinary and enriched order polynomials, and hence by (2.3) and (2.4), the same $W$-polynomials and $\bar{W}$-polynomials.

Note that one may define an acyclic orientation of the covering relation of $P$ by replacing each covering pair $i \prec j$ with the arc $i \leftarrow j$ (if $i<j$ ) or $i \rightarrow j$ (if $i>j$ ). In these terms, two posets are equivalent if and only if there is an orientationpreserving poset isomorphism between them. Since every acyclic orientation of the covering relation arises in this fashion, one sees that there is a one-to-one correspondence between equivalence classes of posets isomorphic to $P$ and $\operatorname{Aut}(P)$ orbits of acyclic orientations of the covering relation of $P$.

Turning to the computational side, the algorithms we use to calculate $W_{P}(t)$ and $\bar{W}_{P}(t)$ are based on (2.3) and (2.4), and have running times that are polynomial functions of the number of order ideals (i.e., downward-closed subposets) of $P$. It is easy to show that a narrow poset whose vertices may be split into chains whose cardinalities are $k$ and $n-k$ has at most $(k+1)(n-k+1)=O\left(n^{2}\right)$ order ideals and $\left(\begin{array}{l}n \\ k\end{array}\right)$ linear extensions. The latter grows exponentially near $k=n / 2$, so these algorithms run in polynomial time on narrow posets and are exponentially faster (in the worst case) than any algorithms based on the definitions of $W_{P}(t)$ and $\bar{W}_{P}(t)$ as sums over $\mathcal{L}(P)$. Note that a general $n$-vertex poset may have as many as $n$ ! linear extensions but at most $2^{n}$ order ideals, so our preferred algorithms are exponentially faster in the (worst) general case as well.

To describe the algorithms, we first introduce the following pair of relations on the set of order ideals of a given partial ordering $P$ of a finite set of integers. Given two order ideals $I$ and $J$ such that $I \subseteq J$, we define

$$
\begin{aligned}
& I \triangleleft J \text { if } i \prec j \text { implies } i<j \text { for all } i, j \in J-I, \\
& I \triangleright J \text { if } i \prec j \text { implies } i>j \text { for all } i, j \in J-I .
\end{aligned}
$$

Thus, if $I \triangleleft J$, then $J-I$ is naturally labeled as a subposet of $P$, and if $I \triangleright J$, then $J-I$ is dually natural. Now define polynomials $Z_{P}(t)$ and $\bar{Z}_{P}(t)$ via the 
recurrences

$$
Z_{P}(t)=\sum_{I \triangleleft P, I \neq P} t Z_{I}(t), \quad \bar{Z}_{P}(t)=\sum_{I \triangleright J \triangleleft P, I \neq P} t \bar{Z}_{I}(t),
$$

and the initial conditions $Z_{\varnothing}(t)=\bar{Z}_{\varnothing}(t)=1$.

The algorithm implicit in the recurrence for $Z_{P}(t)$ involves a quadratic number of polynomial additions and a linear number of coefficient-shifts as a function of the number of order ideals of $P$. On the other hand, the recurrence for $\bar{Z}_{P}(t)$ appears to be cubic in the number of order ideals; however, if we introduce the auxiliary polynomials

$$
A_{P}(t):=\sum_{I \triangleright P, I \neq P} \bar{Z}_{I}(t) \quad\left(A_{\varnothing}(t)=0\right)
$$

then we have

$$
\bar{Z}_{P}(t)=t A_{P}(t)+\sum_{I \triangleleft P, I \neq P} t\left(A_{I}(t)+\bar{Z}_{I}(t)\right),
$$

so the polynomials $A_{P}(t)$ and $\bar{Z}_{P}(t)$ may be computed simultaneously using at most a quadratic number of polynomial additions and a linear number of shifts.

Once $Z_{P}(t)$ or $\bar{Z}_{P}(t)$ has been computed, a change of variable yields $W_{P}(t)$ or $\bar{W}_{P}(t)$ :

Proposition 2.1. If $P$ is a partial ordering of $[n]$, then

(a) $W_{P}(t)=(1-t)^{n} \cdot Z_{P}\left(\frac{t}{1-t}\right)$,

(b) $\bar{W}_{P}(t)=u^{n}(1+u) \cdot \bar{Z}_{P}\left(\frac{1-u}{2 u}\right)$, where $u=\sqrt{1-t}$.

Proof. If $N_{P}(k)$ and $\bar{N}_{P}(k)$ are the coefficients of $t^{k}$ in $Z_{P}(t)$ and $\bar{Z}_{P}(t)$, then

$$
N_{P}(k)=\sum_{I \triangleleft P, I \neq P} N_{I}(k-1), \quad \bar{N}_{P}(k)=\sum_{I \triangleright J \triangleleft P, I \neq P} \bar{N}_{I}(k-1),
$$

for all posets $P$ and all $k>0$, and $N_{P}(0)=\bar{N}_{P}(0)=\delta_{P, \varnothing}$. It follows that $N_{P}(k)$ is the number of surjective maps $f: P \rightarrow[k]$ satisfying $(2.1)$, and $\bar{N}_{P}(k)$ is the number of semi-surjective maps $f: P \rightarrow[2 k]$ satisfying $(2.2)$, where "semi-surjective" means that the range of $f$ includes $2 i-1$ or $2 i$ for each $i=1, \ldots, k$.

On the other hand, by choosing the range of a map first, one sees that

$$
\Omega_{P}(m)=\sum_{k \geqslant 0}\left(\begin{array}{c}
m \\
k
\end{array}\right) N_{P}(k), \quad \bar{\Omega}_{P}(m)=\sum_{k \geqslant 0}\left(\begin{array}{c}
m \\
k
\end{array}\right) \bar{N}_{P}(k),
$$

so (2.3) and the Binomial Theorem imply (a), whereas (2.4) implies

$$
\bar{W}_{P}\left(\frac{4 t}{(1+t)^{2}}\right)=\frac{2(1-t)^{n}}{(1+t)^{n+1}} \cdot \bar{Z}_{P}\left(\frac{t}{1-t}\right) .
$$

In this case, the transformation $t \mapsto(1-u) /(1+u)$ yields (b).

\section{Sums, DuAls, And COMPlEments}

The ordinal sum of two posets $I$ and $J$ with disjoint vertex sets, denoted $I \oplus J$, is the partial ordering of $I \cup J$ formed by the relations of $I$ and $J$, together with the relations $i \prec j$ for all $i \in I$ and $j \in J$. We say that a nonempty poset $P$ is irreducible if it is not expressible as the ordinal sum of proper subposets of $P$; 
every poset is uniquely decomposable as an ordinal sum of zero or more irreducible posets.

The following factorizations are immediate consequences of the observation that the linear extensions of $P \oplus Q$ are the concatenations of linear extensions of $P$ and $Q$.

Proposition 3.1. If $I, J$ and $K=\{i\} \oplus\{j\}$ are disjoint posets, then

(a) $W_{I \oplus\{i\} \oplus J}(t)=t^{-1} W_{I \oplus\{i\}}(t) W_{\{i\} \oplus J}(t)$,

(b) $\bar{W}_{I \oplus K \oplus J}(t)=t^{-1} \bar{W}_{I \oplus K}(t) \bar{W}_{K \oplus J}(t)$.

In addition, if $I \oplus\{i\} \oplus J$ is naturally labeled, then

(c) $W_{I \oplus J}(t)=t^{-1} W_{I}(t) W_{J}(t)$,

(d) $\bar{W}_{I \oplus J}(t)=t^{-1} \bar{W}_{I}(t) \bar{W}_{\{i\} \oplus J}(t)$.

Let us declare $P$ to be an NRZ-poset (or simply $N R Z$ ) if $W_{P}(t)$ has nonreal zeros. Similarly, we say that $P$ is an $\overline{N R Z}$-poset if $\bar{W}_{P}(t)$ has nonreal zeros.

Corollary 3.2. (a) A minimum-sized NRZ-poset has no internal singleton components.

(b) A minimum-sized $\overline{N R Z-p o s e t ~ h a s ~ n o ~ c o n s e c u t i v e ~ i n t e r n a l ~ s i n g l e t o n ~ c o m p o-~}$ nents.

(c) A minimum-sized naturally labeled NRZ-poset is irreducible.

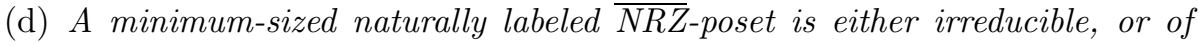
the form $P^{+}$for some irreducible $P$.

Given a partial ordering $P$ of $[n]$, the dual $P^{*}$ is obtained by reversing the relations of $P$ (so $i \prec j$ in $P$ if and only if $i \succ j$ in $P^{*}$ ) and the complement $P_{c}$ is obtained by replacing $i \rightarrow n+1-i$ (so $i \prec j$ in $P$ if and only if $n+1-i \prec n+1-j$ in $P_{c}$ ). Note that $P_{c}$ is isomorphic to $P$, but in general not equivalent to $P$; the acyclic orientation corresponding to $P_{c}$ is obtained by reversing the orientation corresponding to $P$.

The following identities are immediate from the definitions; we omit the proofs.

Proposition 3.3. If $P$ is a partial ordering of $[n]$, then

(a) $W_{P^{*}}(t)=W_{P_{c}}(t)=t^{n+1} W_{P}\left(t^{-1}\right)$,

(b) $\bar{W}_{P^{*}}(t)=\bar{W}_{P}(t)$.

Thus, if $P$ is an NRZ-poset, then so are $P^{*}$ and $P_{c}$, and if $P$ is a naturally labeled

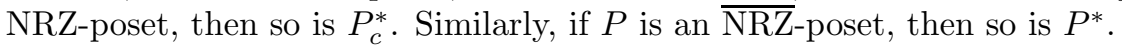

In general, there is no simple relationship between $\bar{W}_{P_{c}}(t)$ and $\bar{W}_{P}(t)$.

\section{Shift-equivalence of Labeled posets}

Let $P$ and $Q$ be partial orderings of [n], and assume temporarily that both posets have maximum and minimum elements. We say that $P$ and $Q$ are shift-equivalent if there is a poset isomorphism $\pi: P \rightarrow Q$ and a shift map $\varepsilon: P \rightarrow \mathbf{Z}$ such that for all covering relations $i \prec j$ in $P$, we have

$$
\varepsilon(j)-\varepsilon(i)=\left\{\begin{array}{cl}
1 & \text { if } i<j \text { and } \pi(i)>\pi(j) \\
-1 & \text { if } i>j \text { and } \pi(i)<\pi(j) \\
0 & \text { otherwise }
\end{array}\right.
$$



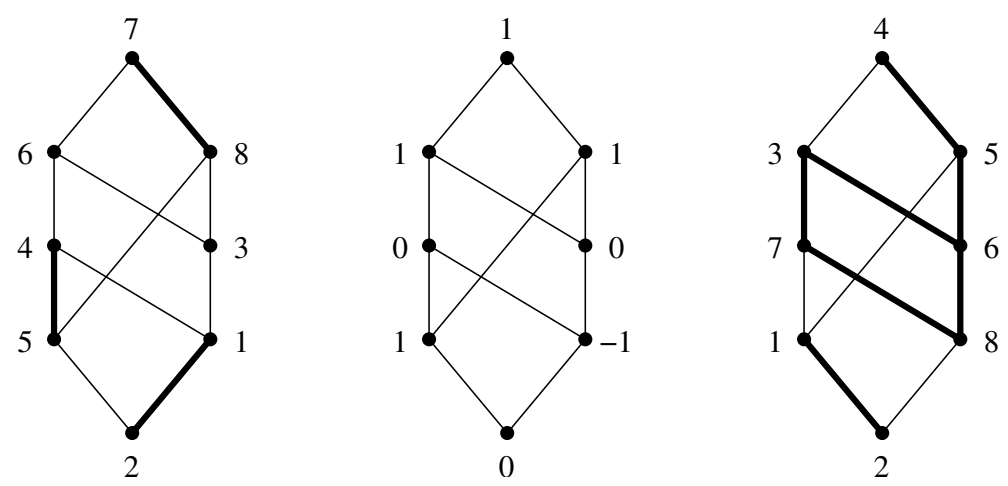

Figure 3. A shift-equivalence of value 1

Note that the shift map $\varepsilon$ is unique up to translation by a constant. Indeed, once $\varepsilon(i)$ is known, then the above relation determines $\varepsilon(j)$ for each $j$ covering $i$ in $P$. The value of the shift is defined to be $\varepsilon\left(1_{P}\right)-\varepsilon\left(0_{P}\right)$, where $0_{P}$ and $1_{P}$ denote the minimum and maximum elements of $P$. The uniqueness of $\varepsilon$ implies that the value of the shift is intrinsic to $\pi$.

An example of two shift-equivalent posets and a corresponding shift map of value 1 appears in Figure 3. (Thick edges indicate covering pairs $i \prec j$ such that $i>j$.)

If $P$ and $Q$ are equivalent, then they are also shift-equivalent via the zero shift map.

Theorem 4.1. (a) Shift-equivalence is an equivalence relation.

(b) If there is a shift-equivalence $P \rightarrow Q$ of value $k$, then $t^{k} W_{P}(t)=W_{Q}(t)$.

Proof. (a) It is clear that shift-equivalence is reflexive. For symmetry, note that if $(\pi, \varepsilon): P \times P \rightarrow Q \times \mathbf{Z}$ is a shift-equivalence from $P$ to $Q$, then $\left(\pi^{-1},-\varepsilon \circ \pi^{-1}\right)$ is a shift-equivalence from $Q$ to $P$. For transitivity, suppose that $(\pi, \varepsilon): P \times P \rightarrow Q \times \mathbf{Z}$ and $(\sigma, \delta): Q \times Q \rightarrow R \times \mathbf{Z}$ are shift-equivalences. We claim that $\gamma=\varepsilon+\delta \circ \pi$ is a valid shift map for the poset isomorphism $\sigma \circ \pi: P \rightarrow R$. Given that $i \prec j$ is a covering relation of $P$, there are eight cases to check, depending on whether $i<j, \pi(i)<\pi(j)$, and $\sigma(\pi(i))<\sigma(\pi(j))$. For example, if $i<j, \pi(i)>\pi(j)$, and $\sigma(\pi(i))<\sigma(\pi(j))$, then $\varepsilon(j)-\varepsilon(i)=1, \delta(\pi(j))-\delta(\pi(i))=-1$, and $\gamma(j)=\gamma(i)$, as required by (4.1). The remaining seven cases are similarly easy and left to the reader.

(b) Let $(\pi, \varepsilon): P \times P \rightarrow Q \times \mathbf{Z}$ be a shift-equivalence of value $k$. Without loss of generality, we may assume that $\varepsilon\left(0_{P}\right)=0$ and $\varepsilon\left(1_{P}\right)=k$. If $f: P \rightarrow[m]$ is one of the maps counted by $\Omega_{P}(m)$ (see (2.1)), we claim that $f^{\prime}=(\varepsilon+f) \circ \pi^{-1}$ is one of the maps counted by $\Omega_{Q}(m+k)$. Indeed, for each covering relation $i \prec j$ in $P$ (or equivalently, $\pi(i) \prec \pi(j)$ in $Q$ ), we see that $i<j$ and $\pi(i)>\pi(j)$ imply $\varepsilon(j)-\varepsilon(i)=1$ and $f(i) \leqslant f(j)$, and hence $f^{\prime}(\pi(i))<f^{\prime}(\pi(j))$. Similarly, $i>j$ and $\pi(i)<\pi(j)$ imply $\varepsilon(j)-\varepsilon(i)=-1$ and $f(i)<f(j)$, and hence $f^{\prime}(\pi(i)) \leqslant f^{\prime}(\pi(j))$. The remaining possibilities are that $i<j$ and $\pi(i)<\pi(j)$, or $i>j$ and $\pi(i)>$ $\pi(j)$; either way, we have $\varepsilon(j)=\varepsilon(i)$, so $f^{\prime}(\pi(i)) \leqslant f^{\prime}(\pi(j))$ in the first case and $f^{\prime}(\pi(i))<f^{\prime}(\pi(j))$ in the second. In particular, having established that $f^{\prime}: Q \rightarrow \mathbf{Z}$ 
is order-preserving, it follows that

$$
1 \leqslant f\left(0_{P}\right)=f^{\prime}\left(0_{Q}\right) \leqslant f^{\prime}(i) \leqslant f^{\prime}\left(1_{Q}\right)=f\left(1_{P}\right)+k \leqslant m+k,
$$

so the range of $f^{\prime}$ is contained in $[m+k]$ and the claim follows. (In particular, this shows that if $m+k<0$, then $f$ cannot exist; i.e., $\Omega_{P}(m)=0$.)

The transformation $f \mapsto f^{\prime}$ is clearly injective, so we have $\Omega_{P}(m) \leqslant \Omega_{Q}(m+k)$ for all integers $m$, following the convention that $\Omega_{P}(-r)=\Omega_{Q}(-r)=0$ for $r>0$. By symmetry, there is also a shift-equivalence $Q \rightarrow P$ of value $-k$, so equality occurs; i.e.,

$$
\Omega_{P}(m)=\Omega_{Q}(m+k)
$$

for all integers $m$. The result now follows from (2.3).

Remark 4.2. (a) If $P$ is a partial ordering of $[n]$ that (possibly) lacks a maximum or minimum element, let $\hat{P}$ denote the partial ordering of $[n+2]$ obtained by renumbering the vertices of $\{0\} \oplus P \oplus\{n+1\}$ via the rule $i \mapsto i+1$. We may define two such posets $P$ and $Q$ to be shift-equivalent if $\hat{P}$ and $\hat{Q}$ are shift-equivalent in the original sense. Bearing in mind that $W_{\hat{P}}(t)=W_{P}(t)$, the above theorem remains valid in this more general setting.

(b) It is easy to check that a poset $P$ is "sign-graded" in the sense of Brändén B2] if and only if $P$ is shift-equivalent to its complement $P_{c}$.

(c) We do not know of an analogue of shift-equivalence for $\bar{W}$-polynomials, although in the theory of enriched $P$-partitions, one knows that $\bar{W}_{P}(t)=\bar{W}_{Q}(t)$ whenever $P$ and $Q$ are "weakly equivalent" (see Section 2.3 of [St]).

(d) A simple operation on equivalence classes of posets that preserves the $\bar{W}$ polynomial may be based on the observation that the peak set of a permutation of $[n]$ is invariant when the elements 1 and 2 are interchanged. If $P$ is a partial ordering of $[n]$, and $i \leftarrow j$ is an arc such that $i$ has outdegree 0 and $j$ has outdegree 1 in the acyclic orientation corresponding to the equivalence class of $P$, then we may renumber the vertices so that $i=1$ and $j=2$ without changing the equivalence class. In that case, interchanging 1 and 2 will reverse the orientation of one arc, but does not change the $\bar{W}$-polynomial.

\section{The STRUCTURE OF NARROW POSETS}

The irreducible narrow posets are especially nice.

Proposition 5.1. If $P$ is irreducible and narrow, then there is a unique partition of the vertices into two chains (or $P$ consists of a single vertex). Furthermore, both chains are necessarily maximal.

Proof. Suppose that $P$ is the union of (nonempty) chains $C_{1}$ and $C_{2}$. The two chains must necessarily be disjoint; otherwise, an element $i$ in their intersection would be comparable to every element of $P$, and $P$ would be the ordinal sum of the subposets formed by $\{j: j \prec i\},\{i\}$, and $\{j: j \succ i\}$. However, at least two of these subposets must be nonempty, contradicting the irreducibility of $P$.

It follows that both chains must be maximal; otherwise, if $C_{1}$ could be enlarged by the addition of an element from $C_{2}$, we could express $P$ as the union of two overlapping chains. In particular, the minimum elements of $C_{1}$ and $C_{2}$ must be incomparable.

Given another partition of $P$ into chains, say $C_{1}^{\prime} \cup C_{2}^{\prime}$, let us relabel them if necessary so that $C_{1}$ and $C_{1}^{\prime}$ share the same minimum element. It cannot be the 
case that $C_{1} \subset C_{1}^{\prime}$, or $C_{1}$ would not be maximal. Hence, there must be a covering pair $i_{1} \prec j_{1}$ in $C_{1}$ such that $i_{1} \in C_{1}^{\prime}$ and $j_{1} \in C_{2}^{\prime}$. The next element $j_{2}$ above $i_{1}$ in $C_{1}^{\prime}$ and the next element $i_{2}$ below $j_{1}$ in $C_{2}^{\prime}$ must therefore both be elements of $C_{2}$ (and both exist, or else either the maximum or minimum elements of $C_{1}^{\prime}$ and $C_{2}^{\prime}$ would be comparable.)

We must also have $i_{2} \prec j_{2}$; otherwise, $i_{1} \prec j_{2} \prec i_{2} \prec j_{1}$ and we contradict the fact that $C_{1}$ is maximal. Furthermore, the relation $i_{2} \prec j_{2}$ must be a cover; otherwise, if $i_{2} \prec i \prec j_{2}$, then either $i \prec i_{1}$ (if $i \in C_{1}^{\prime}$ ) or $j_{1} \prec i$ (if $i \in C_{2}^{\prime}$ ), so either $i_{2} \prec i \prec i_{1} \prec j_{1}$ (contradicting the maximality of $C_{2}^{\prime}$ ) or $i_{1} \prec j_{1} \prec i \prec j_{2}$ (contradicting the maximality of $C_{1}^{\prime}$ ). Thus, each of $i_{1}$ and $i_{2}$ are covered by $j_{1}$ and $j_{2}$, so $P$ is the ordinal sum of the subposets formed by $\left\{j: j \preccurlyeq i_{1}\right.$ or $\left.j \preccurlyeq i_{2}\right\}$ and $\left\{j: j \succcurlyeq j_{1}\right.$ or $\left.j \succcurlyeq j_{2}\right\}$, a contradiction.

Remark 5.2. Although it will not be needed in what follows, it is noteworthy that the number of isomorphism classes of irreducible narrow posets with $n>1$ vertices is

$$
\begin{array}{cl}
(1 / 2) C_{n-1} & \text { if } n \text { is odd, } \\
(1 / 2)\left(C_{n-1}+C_{(n-2) / 2}\right) & \text { if } n \text { is even, }
\end{array}
$$

where $C_{n}=(2 n) ! /(n+1) ! n$ ! denotes the $n$th Catalan number. Consequently, the generating series for isomorphism classes of all narrow posets is $(1-x / 2-I(x)-$ $\left.I\left(x^{2}\right)\right)^{-1}$, where $I(x)=(1 / 2) \sum_{n \geqslant 1} C_{n-1} x^{n}=(1-\sqrt{1-4 x}) / 4$. (See Exercise 6.45 of EC2.)

We define an irreducible narrow poset to be of type $(m, n)$ if the chains in the unique two-chain partition of the vertices have cardinalities $m \geqslant n \geqslant 0$. In particular, a singleton has type $(1,0)$. More generally, we say that a narrow poset has type $\left(\left(m_{1}, n_{1}\right), \ldots,\left(m_{l}, n_{l}\right)\right)$ if its irreducible components have these types (in this order).

Corollary 5.3. If $P$ is irreducible and narrow, then $P$ has at most one nontrivial automorphism; in that case, $P$ is necessarily of type $(m, m)$ for some $m$, and the automorphism interchanges the members of the chains in the two-chain partition of $P$.

If $P$ is irreducible of type $(m, n)$, then (up to isomorphism) we may assume that the unique two-chain partition of $P$ is given by

$$
1 \prec 2 \prec \cdots \prec m, \quad m+1 \prec m+2 \prec \cdots \prec m+n,
$$

and the remaining covering relations $i \prec j$ in $P$ involve pairs $(i, j) \in C_{m, n}$, where

$$
\begin{aligned}
C_{m, n}:=\{(i, j): 1 \leqslant i<m, m+1<j \leqslant m+n\} & \\
& \cup\{(i, j): m+1 \leqslant i<m+n, 1<j \leqslant m\} .
\end{aligned}
$$

Furthermore, since any subset of the covering relation of a poset is the covering relation of another poset, it follows that the set of irreducible narrow posets whose chain partition is (5.1) may be viewed as a simplicial complex $\Delta(m, n)$ over the ground set $C_{m, n}$.

More generally, fix a type $\mu=\left(\left(m_{1}, n_{1}\right), \ldots,\left(m_{l}, n_{l}\right)\right)$ and a poset $P_{\mu}=P_{1} \oplus$ $\cdots \oplus P_{l}$, where $P_{i}$ is a disjoint union of chains of type $\left(m_{i}, n_{i}\right)$ (or a singleton, if $\left.\left(m_{i}, n_{i}\right)=(1,0)\right)$. A consequence of Proposition 5.1 is that every narrow poset of 
type $\mu$ has a unique subposet isomorphic to $P_{\mu}$, and by (again) identifying posets with their covering relations, one sees that the product complex

$$
\Delta(\mu)=\Delta\left(m_{1}, n_{1}\right) \times \cdots \times \Delta\left(m_{l}, n_{l}\right)
$$

is the simplicial complex of all narrow posets of type $\mu$ that contain $P_{\mu}$ as a subposet. Moreover, the number of times a given isomorphism class of narrow posets of type $\mu$ is represented in $\Delta(\mu)$ is the index of the stabilizer subgroup of this class in $\operatorname{Aut}\left(P_{\mu}\right)$; this latter group has order $2^{k}$, where $k=k(\mu):=\left|\left\{i: m_{i}=n_{i}\right\}\right|$.

Now define a rooted tree structure on $\Delta(\mu)$ by declaring $P_{\mu}$ to be the root object, and for all other posets $P$ in $\Delta(\mu)$, the parent of $P$ is defined to be the poset whose covering relation is obtained by deleting the first covering relation in $P$ that is not in $P_{\mu}$, relative to some fixed total ordering of the ground set of potential covering pairs.

We are able to efficiently search through the narrow posets of type $\mu$ by implementing a depth-first search through this spanning tree. The main technical issue for any depth-first search of a tree that is too large to be stored in its entirety is the problem of generating the children of a given object from local data. In our case, this is relatively easy: after arriving at a poset $P$ for the first time, we generate the list of all potential covering pairs $(i, j)$ that precede the first covering pair of $P$ in the chosen total order and check to see whether the relation $i \prec j$,

1. conflicts with any existing relation of $P$,

2. would destroy the covering status of some existing relation in $P$, or

3. would destroy the irreducibility of some existing component of $P$.

The list of pairs that survive tests 1-3 correspond to the children of $P$; we add this list to a stack of lists. If the child-list of $P$ is nonempty, we delete the first item on it, move to the corresponding poset, and continue the search; if the child-list is empty, we delete it from the stack, backtrack to the parent of $P$, and continue deleting and backtracking until we reach an ancestor with a nonempty child-list. We then delete the first item on its child-list, move to the corresponding poset, and resume the search. The search ends when the stack is empty.

To avoid processing isomorphic copies of the same poset during a search of $\Delta(\mu)$, we identify in advance a set of generators for $\operatorname{Aut}\left(P_{\mu}\right)$. If a given poset $P$ is not the lexicographically first member of its $\operatorname{Aut}\left(P_{\mu}\right)$-orbit, then we may skip any testing of $P$. This requires comparing $P$ against at most $k(\mu)$ other posets; often, $k(\mu)$ will be 0 .

\section{THE SEARCH RESULTS}

Recall from Section 2 that the equivalence classes of labelings of a given poset $P$ correspond to acyclic orientations of the covering relation. Furthermore, the labeling of $P$ influences the computation of $W_{P}(t)$ and $\bar{W}_{P}(t)$ only through the relations $\triangleright$ and $\triangleleft$ on the order ideals of $P$, and it is easy to recover $\triangleright$ and $\triangleleft$ from a given acyclic orientation. Thus, to test whether some relabeling of $P$ is (say) an NRZ-poset, we first generate in advance the list of all order ideals of $P$, and run a search through all orientations of the covering relation of $P$, rejecting those orientations that produce cycles. Each acyclic orientation induces relations $\triangleright$ and $\triangleleft$ on the pre-stored set of order ideals, from which we compute the corresponding $W$-polynomial via the algorithm of Section 2, and test the result for nonreal zeros using Sturm's Theorem (e.g., see [K, p. 416]). Note that for NRZ-posets, we may 

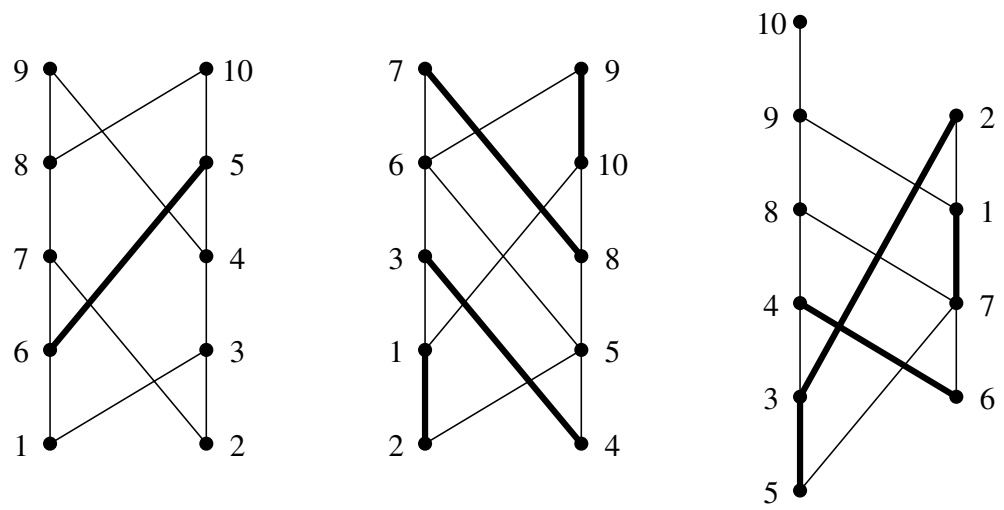

FiguRE 4. Minimal narrow NRZ-posets.

save a factor of 2 by fixing the orientation of one covering pair in $P$, relying on the fact that the complement of an NRZ-poset is also NRZ (Proposition 3.3).

With this in mind, we searched for a minimum-sized narrow NRZ-poset on $n$ vertices for each $n \leqslant 10$ (roughly) as follows. First, we generated the type vectors of all narrow $n$-vertex posets, discarding those types $\mu$ that have internal singletons (Corollary 3.2(a)), as well as those for which the dual type precedes $\mu$ in lexicographic order. (By Proposition 3.3, one need not check both $P$ and $P^{*}$.) We then ran a search through the isomorphism classes of narrow posets of the remaining types via the algorithm described in the previous section. For each isomorphism class representative $P$, we then searched for NRZ-posets among the relabelings of $P$ as above.

According to the results of the search, there are exactly 48 equivalence classes of narrow NRZ-posets on 10 vertices, and none smaller. Of these, there are 24 classes up to complementation (i.e., no equivalence class is self-complementary). These 24 classes may be grouped into four shift-equivalence classes, in two of which the underlying poset is self-dual. Representatives from three of the shift-equivalence classes are displayed in Figure 4; the dual of the one on the right represents the fourth class. From left to right, their $W$-polynomials are

$$
\begin{gathered}
11 t^{2}+42 t^{3}+50 t^{4}+18 t^{5}+t^{6}, \quad 11 t^{3}+41 t^{4}+46 t^{5}+14 t^{6}+t^{7} \\
12 t^{3}+39 t^{4}+35 t^{5}+5 t^{6} .
\end{gathered}
$$

We then searched for minimum-sized NRZ-posets on $n \leqslant 11$ vertices by essentially the same method, the main modification being that we may discard only those type vectors $\mu$ that include two consecutive internal singletons (Corollary 3.2(b)). Note also that since the $\bar{W}$-polynomial of a poset and its complement are unrelated in general, we may not fix in advance the orientation of an arbitrarily chosen covering pair.

In this case, we found that there are 10 equivalence classes of narrow $\overline{\mathrm{NRZ}}$-posets with 11 vertices, and none smaller. Of these, there are five classes up to duality, but only two distinct $\bar{W}$-polynomials occur among these five classes. Representatives from two of the classes are displayed in Figure 5 , and their $\bar{W}$-polynomials (in 

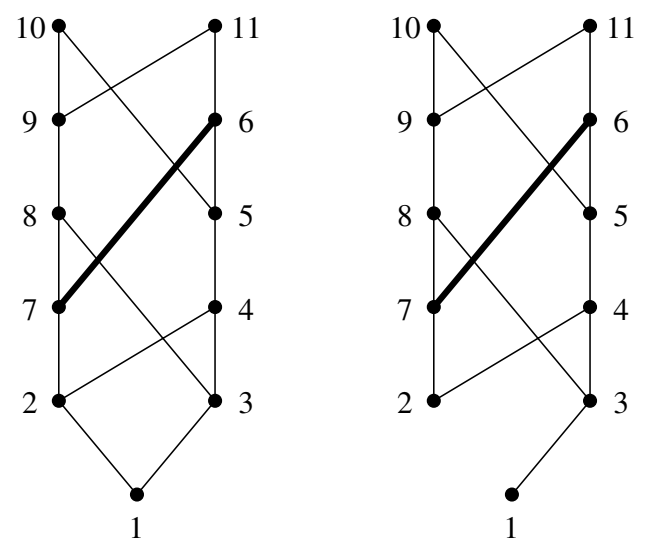

Figure 5. Minimal narrow NRZ-posets

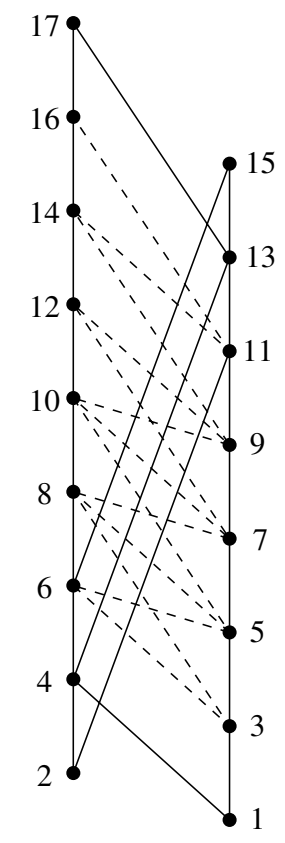

FiguRE 6 . The 17-vertex narrow NRZ-posets, superimposed

displayed order) are:

$$
11 t^{2}+42 t^{3}+50 t^{4}+18 t^{5}+t^{6}, \quad 23 t^{2}+82 t^{3}+89 t^{4}+27 t^{5}+t^{6} .
$$

Representatives of the remaining classes may be obtained (up to duality) by interchanging vertices 1 and 3 in both examples, or by interchanging 1 and 2 in the first example; the fact that these interchanges preserve the $\bar{W}$-polynomial is explained in Remark 4.2(d).

For naturally labeled posets, the search constraints are considerably simpler: the natural labelings of a poset form a single equivalence class, so we need to compute only one $W$-polynomial or $\bar{W}$-polynomial for each isomorphism class. Furthermore, 
by Corollary 3.2(c), we may restrict the search for a minimum-sized naturally labeled narrow NRZ poset to those that are irreducible. We may also restrict the analogous search for NRZ-posets to irreducible types, bearing in mind that by Proposition 1.1 and Corollary 3.2(d), this omits potentially minimal examples of the form $P^{+}$, where $P$ is NRZ.

We found that there are 58 isomorphism classes of narrow posets on 17 vertices whose natural labelings are NRZ, and none smaller. These isomorphism classes may be naturally divided into two groups of 29 so that: (1) the posets within each group are all closely related to each other, and (2) the two groups are dual to each other. Figure 6 illustrates the similarity within one of the two groups: there are representatives from each of the 29 posets in this class that include all of the relations indicated by solid edges, and the remaining covering relations in each of the posets involve $2,3,4$, or 5 of the dashed edges. For example, the poset in Figure 1 is isomorphic to the poset generated by the relations $3 \prec 6,5 \prec 8,7 \prec 10$, $9 \prec 12$, and $11 \prec 14$, together with the solid-edge relations in Figure 6. A complete list of these posets and their $W$-polynomials is available at the web site mentioned in the introduction.

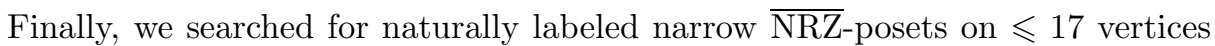
and found none, so we conclude that there are no naturally labeled narrow NRZposets that are smaller than the 58 one obtains by adjoining minimum elements to the naturally labeled narrow NRZ-posets on 17 vertices. However, there could also be naturally labeled narrow NRZ-posets on 18 vertices that are not of this form.

\section{Future DireCtions}

A number of interesting questions remain.

1. Brändén [B2] has shown that sign-graded posets (and hence all naturally labeled graded posets) have $W$-polynomials that are symmetric and unimodal. Are all of the zeros of these polynomials real?

2. Are the coefficients of $W_{P}(t)$ and $\bar{W}_{P}(t)$ unimodal? log-concave? Note that every polynomial whose zeros are real and nonpositive has log-concave (and hence unimodal) coefficients, so any counterexamples would have to be NRZ or NRZ. It would be natural to search among the narrow posets for counterexamples, although we expect that if such posets exist, they will be much larger than the NRZ and

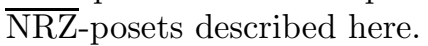

3. If $P$ is naturally labeled, then the polynomials $Z_{P}(t)$ and $\bar{Z}_{P}(t)$ of Section 2 are of independent interest. Indeed, $Z_{P}(t)$ is a generating function for chains in the lattice of order ideals of $P$, and Hsiao (see Section 5.3 of $[\mathrm{H}]$ ) has shown that $\bar{Z}_{P}(t)$ is a chain-enumerator for a poset of "signed" order ideals of $P$. Are the coefficients of $Z_{P}(t)$ and $\bar{Z}_{P}(t)$ unimodal? log-concave? In the naturally labeled case, Björner and Farley $[\mathrm{BF}]$ have recently shown that at least $3 / 4$ of the inequalities necessary for the unimodality of $Z_{P}(t)$ are valid, and Hsiao $[\mathrm{H}]$ notes that the same is true for $\bar{Z}_{P}(t)$.

We remark that it is well known (and easy to see from Proposition 2.1) that all zeros of $Z_{P}(t)$ are real if and only if $W_{P}(t)$ has the same property, and there is a similar relationship between $\bar{Z}_{P}(t)$ and $\bar{W}_{P}(t)$. Also, by a theorem of Brenti (see Theorem 2.5.8 of $[\mathrm{Br}]$ ) it follows that if the coefficients of $W_{P}(t)$ are log-concave, then the same holds for $Z_{P}(t)$. 


\section{REFERENCES}

[BF] A. Björner and J. D. Farley, Chain polynomials of distributive lattices are $75 \%$ unimodal, Electron. J. Combin. 12 (2005), Note 4, 7 pp. MR2134190 (2005m:06004)

[B1] P. Brändén, On operators on polynomials preserving real-rootedness and the NeggersStanley Conjecture, J. Algebraic Combin. 20 (2004), 119-130. MR.2104673 (2005h:12004)

[B2] P. Brändén, Sign-graded posets, unimodality of $W$-polynomials and the Charney-Davis Conjecture, Electron. J. Combin. 11(2) (2005), R9. MR2120105 (2005k:06006)

[B3] P. Brändén, Counterexamples to the Neggers-Stanley conjecture, Electron. Res. Announc. Amer. Math. Soc. 10 (2004), 155-158. MR2119757 (2005j:06002)

[Br] F. Brenti, Unimodal, log-concave and Polya frequency sequences in combinatorics, Mem. Amer. Math. Soc. (1989), no. 413. MR0963833 (90d:05014)

[BM] G. Brinkmann and B. McKay, Posets on up to 16 points, Order 19 (2002), 147-179. MR 1922916 (2003e:05002)

[G] V. Gasharov, On the Neggers-Stanley conjecture and the Eulerian polynomials, J. Combin. Theory Ser. A 82 (1998), 134-146. MR.1620853 (99i:05020)

[H] S. K. Hsiao, A signed analog of the Birkhoff transform, J. Combin. Theory Ser. A 113 (2006), no. 2, 251-272. MR2199274

[K] D. E. Knuth, "The Art of Computer Programming, Vol. 2: Seminumerical Algorithms," (2nd ed.), Addison-Wesley, Reading MA, 1981. MR.0633878 (83i:68003)

[N] J. Neggers, Representations of finite partially ordered sets, J. Combin. Inform. System Sci. 3 (1978), 113-133. MR0551484 (58:27668)

[RW] V. Reiner and V. Welker, On the Charney-Davis and Neggers-Stanley Conjectures, J. Combin. Theory Ser. A 109 (2005), 247-280. MR2121026 (2006e:06003)

[Si] R. Simion, A multi-indexed Sturm sequence of polynomials and unimodality of certain combinatorial sequences, J. Combin. Theory Ser. A 36 (1984), 15-22. MR.0728500(85e:05015)

[S] R. P. Stanley, Ordered structures and partitions, Mem. Amer. Math. Soc. (1972), no. 119. MR0332509 (48:10836)

[EC2] R. P. Stanley, "Enumerative Combinatorics, Vol. 2," Cambridge Univ. Press, Cambridge, 1999. MR.1676282 (2000k:05026)

[St] J. R. Stembridge, Enriched P-partitions, Trans. Amer. Math. Soc. 349 (1997), 763-788. MR.1389788 (97f:06006)

[W] D. G. Wagner, Enumeration of functions from posets to chains, European J. Combin. 13 (1992), 313-324. MR.1179527(94c:05008)

Department of Mathematics, University of Michigan, Ann Arbor, Michigan 481091109

E-mail address: jrs@umich.edu 